\title{
Study on Hybrid Characteristics of Medicinally Used Cultivated Codonopsis Species Using Ribosomal Internal Transcribed Spacer (ITS) Sequencing
}

\author{
Li-Jun Liang ${ }^{1,2,3}$, Er-Huan Wang ${ }^{4}$, Yi-Chen Yang ${ }^{4}$, Bing-Cong Xing ${ }^{1,3}$, Wei Ji ${ }^{5}$, Feng Liu ${ }^{4}$ and \\ Zong-Suo Liang $1,3,6,7$,* \\ 1 Institute of Soil and Water Conservation, CAS \& MWR, Yangling 712100, China; llj@zafu.edu.cn (L.-J.L.); \\ xingbingcong@163.com (B.-C.X.) \\ 2 College of Landscape Architecture, Zhejiang Agriculture \& Forestry University, Hangzhou 311300, China \\ 3 University of Chinese Academy of Sciences, Beijing 100049, China \\ 4 Buchang Pharmaceuticals Co., Ltd., Xi'an 712000, China; jilinweh@163.com (E.-H.W.); \\ loftyideals@163.com (Y.-C.Y.); liufeng1720@163.com (F.L.) \\ 5 College of Horticulture, Shanxi Agricultural University, Taigu 030801, China; jiweiputao@163.com \\ 6 Key Laboratory of Plant Secondary Metabolism and Regulation of Zhejiang Province, College of Life \\ Sciences, Zhejiang Sci-Tech University, Hangzhou 310018, China \\ 7 College of Life Sciences, Northwest A\&F University, Yangling 712100, China \\ * Correspondence: liangzs@ms.iswc.ac.cn; Tel.: +86-0571-8684-3684
}

Received: 11 June 2018; Accepted: 26 June 2018; Published: 28 June 2018

\begin{abstract}
Codonopsis taxa, as a traditional Chinese medicinal and edible plant, has found expanding domestic and foreign applications in recent decades. However, the poor management in germplasm resources market has inevitably caused an unnecessary hybrid of the provenances. In order to clarify the hybrid characteristics of germplasm resources in the main production area, the Codonopsis cultivars collected from the provinces Gansu, Shannxi, Shanxi, and Hubei of China were researched, using internal transcribed spacer (ITS) sequence technology. The confirmation of additive nucleotides based on the ITS sequencing of polymerase chain reaction (PCR) mixture was optimized and used to study the hybrid of Codonopsis cultivars. The results showed that when the ratio of PCR mixture increased up to 15 percent, the presence of a double peak in the sequencing electrophoresis map could be confirmed, suggesting the existence of additive nucleotides. According to the method above, 46 samples of Codonopsis cultivars collected during 2016 and 2017 were studied and compared with the samples collected from the year 2009 to 2010. All of the samples collected during 2016 and 2017 were hybridized and no genetic pure lines were found. In addition, the sites of variable base reduced greatly, concentrating at positions 122 and/or 226. These phenomena suggested that the genetic diversity of Codonopsis cultivars declined and the germplasm resources gradually converged. More attention should be paid to the reasonable exploitation and genetic breeding of Codonopsis taxa.
\end{abstract}

Keywords: Codonopsis taxa; ITS; additive nucleotide; hybrid

\section{Introduction}

Codonopsis Radix, normally called 'Dangshen', is prescribed as the dried root of Codonopsis pilosula (Franch.) Nannf., C. pilosula Nannf. var. modesta (Nannf.) L. D. Shen, and C. tangshen Oliv. of Campanulaceae [1]. Codonopsis Radix is a traditional Chinese medicinal and edible plant, strengthening the immune system, replenishing qi (vital energy), improving poor gastrointestinal function, and lowering blood pressure [1]. With a history of more than 300 years, Codonopsis taxa is mainly distributed in four provinces of China, including Gansu, Shannxi, Shanxi, and Hubei. 
Since 2006, the increased demand in the domestic and international market stimulated large-scale and regional cultivation of Codonopsis taxa, especially in Gansu province, accounting for $90 \%$ of the total cultivation area in China [2]. Because of the lack of professional support in seed and seedling production, most farmers buy seeds and seedlings only in pursuit of high yield. C. pilosula in Min County and C. pilosula var. modesta in Longnan city of Gansu Province are always selected as the germplasm resources owing to the high yield. Such a production model probably resulted in homogenization of germplasm resources and a decrease in genetic diversity [3-5]. In this study, DNA molecular markers were used to analyze the germplasm resources and genetic characteristics of Codonopsis taxa in the main production areas, and the hybrid characteristics of cultivated species were elucidated.

A large number of molecular marker techniques, such as simple sequence repeat (SSR), single nucleotide polymorphism (SNP), and internal transcribed spacer (ITS), have been used in plant classification, identification, and analysis of genetic characteristics [6-15]. Among them, ITS sequence of nuclear ribosomal DNA (nrDNA) has been proved to be a helpful non-coding marker to infer hybridization events [5,16-22]. Because ITS has relatively high levels of phylogenetically informative sequence variation [23], it can provide evidence of evolution when the hybrid retains the repeat types contributed from each parental species [24-26]. Additive nucleotides (also called variable base) presented in ITS sequences are often used to study the origin of hybridization parents [27-41]. They are formed from two or more different bases that appear simultaneously at one sequence position, and each of them represents a single parent and is heterozygous at that position. However, because of the influence of background noise from electrophoresis sequencing, it is critical to determine whether variable bases are actually present. To determine the existence of variable bases, the intensity of the primary signal, secondary signal, and noise signal of variable bases shown in the electrophoretic sequencing map are calculated and analyzed statistically.

Although molecular marker techniques have been widely used in studying the diversity of medicinal plants, ITS and SSR were used to study the diversity of Codonopsis taxa $[4,5,19]$. He JY et al. [19] studied the origin of Codonopsis hybrids, as well as the hybrid characteristics of Codonopsis collected during the year 2009 and 2010, based on the ITS gene sequence. Large-scale plantation of Codonopsis cultivars in the main production area after the year 2010 probably accelerated the hybrid of germplasm resources. To reveal the changes in Codonopsis cultivars after seven years and arouse the attention on protecting the genetic diversity of Codonopsis taxa, this study sequenced the ITS genes of 46 samples of Codonopsis cultivars from the provinces Gansu, Shaanxi, Shanxi, Hubei, and Ningxia Hui Autonomous Region of China collected during the year 2016 and 2017.

\section{Results and Discussion}

\subsection{Verification of Additive Nucleotides}

To distinguish the secondary peak at the 122nd position from noise and verify the additive nucleotides, the secondary peak $(S)$-value and background noise level $(N)$-value were calculated according to Equations (1) and (2). Table 1 presents the $S$-value of the additive peak at the 122nd position and the $N$-value of the high noise near the 122nd position in the ITS gene $(n=5)$. When the percentage of polymerase chain reaction (PCR) product of CT46 (pCT46) increased from $10 \%$ to $50 \%$, the average $S$-value rose from $11.54 \%$ to $47.72 \%$. The $S$-value of the secondary peak at the 122 nd position was positively correlated to the amount of pCT46. Variance analysis between the $S$-value and $\mathrm{N}$-value was performed and the significance of the difference between the two values was calculated to prove the existence of the double peaks at this position. Correspondingly, the existence of the double peaks suggested the presence of additive nucleotides. The results in Table 1 indicated that when the amount of pCT46 was $10 \%$ or less, the difference between the $S$-value and $N$-value was insignificant, and the secondary peak could not be confirmed, as well as the presence of additive nucleotides. When the amount of pCT46 increased from $10 \%$ to $15 \%$, the existence of additive 
nucleotides should be detected by the significant of the difference between the $S$-value and $N$-value. When the amount of pCT46 was 15\% or more, the existence of additive nucleotides could be confirmed directly. This phenomenon was also detected by Kitani Y. et al. [17] and He JY. et al. [19]. Because the $S$-value of the secondary peak was similar to the amount of the PCR product mixture, it could be used to calculate the hybrid rate.

Table 1. $S$-value of the additive peak at the 122nd Position and $N$-value of the high noise sites near the 122 nd position in the internal transcribed spacer (ITS) gene $(n=5)$.

\begin{tabular}{ccccc}
\hline pCT46 (\%) $^{a}$ & Main Signal & S-Value (\%) & $N$-Value (\%) & Significance $^{b}$ \\
\hline 10 & C & $11.54 \pm 1.46$ & $10.82 \pm 0.98$ & \\
15 & C & $14.82 \pm 1.16$ & $10.64 \pm 1.13$ & $* *$ \\
20 & C & $21.08 \pm 2.23$ & $10.66 \pm 1.07$ & $* *$ \\
30 & C & $29.14 \pm 1.65$ & $8.92 \pm 1.18$ & $* *$ \\
40 & C & $39.60 \pm 1.87$ & $9.02 \pm 1.19$ & $* *$ \\
50 & C & $47.72 \pm 1.37$ & $8.84 \pm 1.16$ & $*$ \\
\hline
\end{tabular}

${ }^{a}$ indicates the percent of polymerase chain reaction (PCR) products of sample CT46 to sample CP02. ${ }^{b}$ indicates the significance of difference between $S$-value and $N$-value. The $S$-value and $N$-value were equal to 'Average \pm S.D.'. The double asterisk indicates the difference analyzed between the $S$-value and $N$-value was very significant $(p<0.01)$.

\subsection{ITS Sequences of Codonopsis Plant Species}

The length of the ITS (ITS1-5.8S-ITS2) region was $655 \mathrm{bp}$ in the selected 46 Codonopsis specimens, and the length of ITS1, 5.8S, and ITS2 was 257, 163, and $235 \mathrm{bp}$, respectively (Figure S1). When comparing the ITS sequences of C. pilosula, C. pilosula var. modesta, and C. tangshen in this study with those of the genus Codonopsis in GenBank online, high homology was found with accession numbers EF190460, EF190461, EF190462, and AB769272 (designated as P0, PM0, T0, and S0), which had been reported by Lin TC. et al. [16] and He JY. et al. [19] as the pure line. Among the four species, AB769272 is the ITS sequence of an unidentified specimen of genus Codonopsis. As shown in Table 2, the ITS sequences showed different variable sites in C. pilosula, C. pilosula var. modesta, and C. tangshen. One or two positions including the 122nd and/or 226th positions only appeared on ITS1 regions of C. pilosula and C. pilosula var. modesta, while the 135th and 500th positions presented on C. tangshen. The variable sites on all of these specimens were additive bases $Y(Y=C$ and $T)$ or $R(R=A$ and $\mathrm{G})$. The additive nucleotides $Y$, representing double peaks of $C$ and $T$ in electrophoretogram, were frequently observed at positions 122 and/or 226, especially at the former. Among them, the additive nucleotides $\mathrm{Y}(\mathrm{T}>\mathrm{C})$ at the 122nd position accounted for a large proportion, with only four specimens, including $\mathrm{CP} 26, \mathrm{CP} 34, \mathrm{CP} 37$, and $\mathrm{CP} 38$ displaying $\mathrm{Y}(\mathrm{C}>\mathrm{T})$ at the same position. At the 226th position, all the specimens presented additive nucleotides $\mathrm{Y}(\mathrm{C}>\mathrm{T})$, except for the $\mathrm{CP} 10(\mathrm{~T}>\mathrm{C})$ (Figure 1).

According to the composition of the nucleotide in the ITS sequences, the 46 specimens were classified into nine ITS-type sequences. The nine ITS-type and four pure-line sequences were also used to construct the phylogenetic tree in 2.3. The tested C. pilosula was divided into five types, namely, P1, P2, P3, P4, and P5, the corresponding numbers of which were 33, 1, 1, 2, and 2, respectively. C. pilosula var. modesta was divided into three types according to the method above, namely, PM1, PM2, and PM3, with the numbers of 4, 1, and 1, respectively. Only one C. tangshen specimen (CT46) was found in this study.

As one of the main production areas of Codonopsis cultivars, Gansu province of China has two species, including C. pilosula and C. pilosula var. modesta. No genetic pure line of them collected during the year 2016 to 2017 had been found according to the ITS sequencing results. There were only five types of ITS sequences in the C. pilosula, and three types in the C. pilosula var. modesta. The variable base concentrated at the 122nd and/or 226th nucleotide sites, and all were located at ITS1 region. In other words, there were no variable base sites in the 5.8S and ITS2 regions. As for the ITS type of the C. pilosula, P1 types were dominant with a percentage near $85 \%$, and for the C. pilosula var. 
modesta, PM1 approached 67\%. Although the C. pilosula is different from C. pilosula var. modesta in morphological characteristics, the ITS sequences of P1 and PM1 were similar, which could not be used to distinguish the two species.

However, in 57 samples tested in the studies of He JY. et al. [19] during the year 2009 and 2010, genetic pure lines of C. pilosula and C. pilosula var. modesta were found and accounted for $13 \%$ and $47 \%$, respectively. ITS type was 11 for C. pilosula and 5 for C. pilosula var. modesta. They also reported that six variable base sites presented in ITS sequences of C. pilosula (122nd, 130th, 226th, 441st, 489th, and 519th) and C. pilosula var. modesta (130th, 226th, 441st, 489th, 509th, and 533rd), while in our research, only the 122nd and 226th were found. Above all, the ITS type of cultivated Codonopsis taxa decreased, as well as the number of the variable base, which suggested a reduction of genetic diversity of the Codonopsis taxa. These phenomena might be related to the gradual convergence of the seedling sources and natural hybridation in the main production areas of Gansu province of China.

Table 2. Types of ITS sequences of Codonopsis species and the assumed lineages related to hybridization.

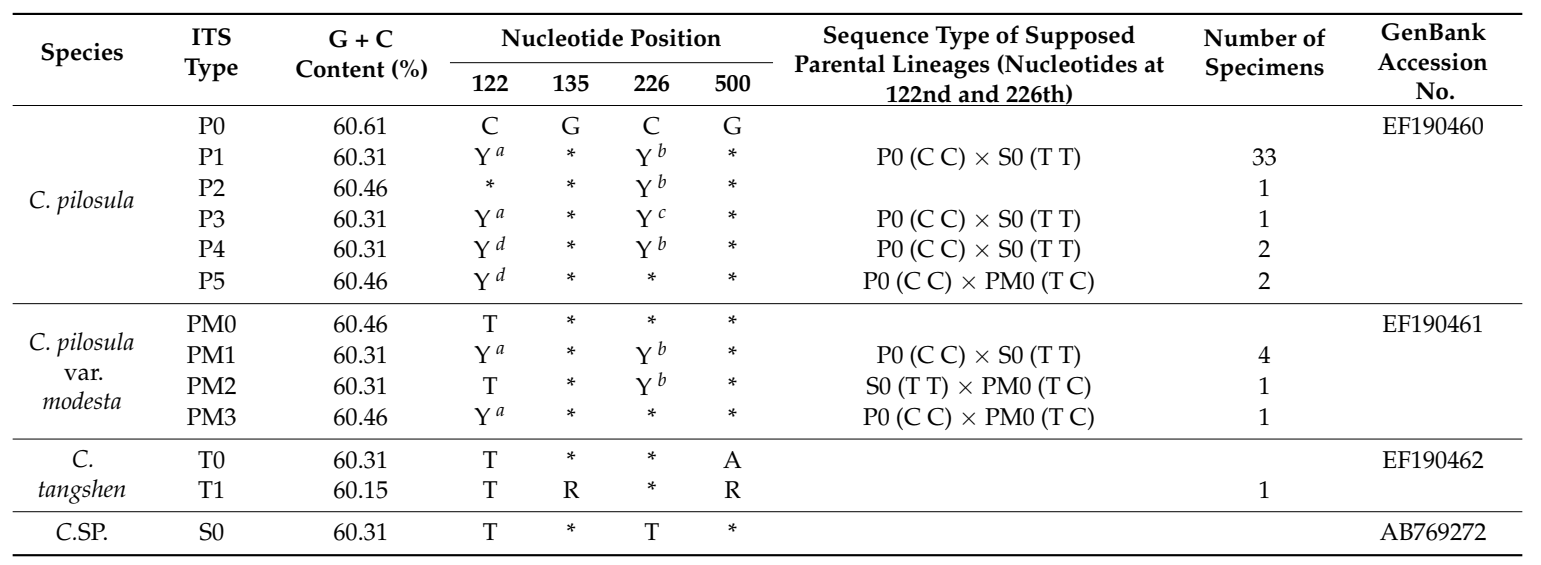

Numerals above sequence are aligned nucleotide positions. $\mathrm{Y}=\mathrm{C}$ and $\mathrm{T}, \mathrm{R}=\mathrm{A}$ and $\mathrm{G}$, asterisk indicates the identical nucleotide to $\mathrm{P} 0 .{ }^{a}$ indicates the additive nucleotide $\mathrm{Y}(\mathrm{T}>\mathrm{C}){ }^{b}$ indicates the additive nucleotide $\mathrm{Y}(\mathrm{C}>\mathrm{T})$; ${ }^{c}$ indicates the additive nucleotide $\mathrm{Y}(\mathrm{T}>\mathrm{C}) ;{ }^{d}$ indicates the additive nucleotide $\mathrm{Y}(\mathrm{C}>\mathrm{T})$.

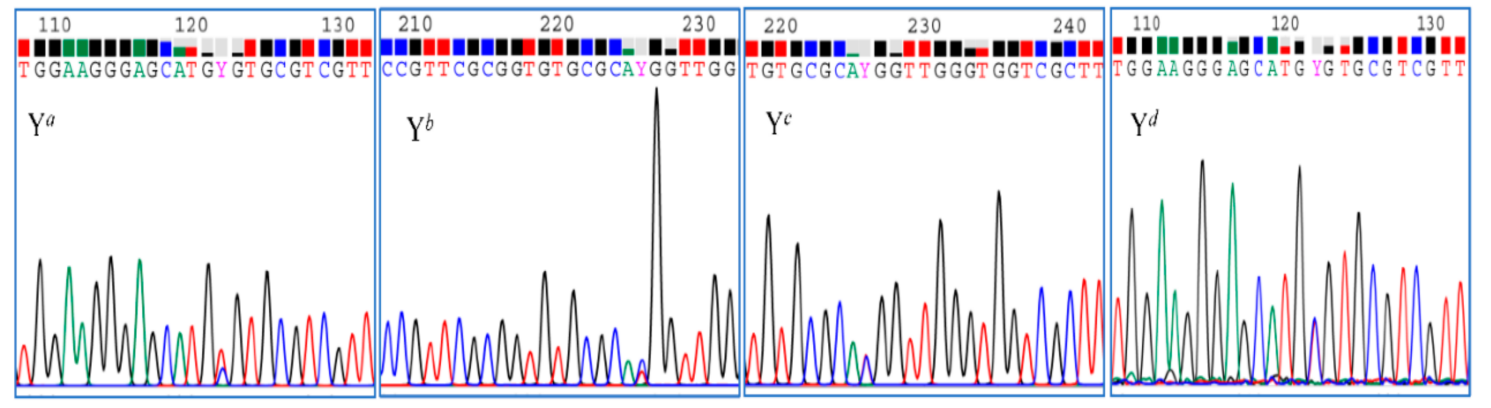

Figure 1. Four types of additive nucleotide $Y$ at position 122nd or 226th. $Y^{a}$ indicates the additive nucleotide $\mathrm{Y}(\mathrm{T}>\mathrm{C})$ at the 122 nd position; $\mathrm{Y}^{b}$ indicates the additive nucleotide $\mathrm{Y}(\mathrm{C}>\mathrm{T})$ at the 226th position; $\mathrm{Y}^{c}$ indicates the additive nucleotide $\mathrm{Y}(\mathrm{T}>\mathrm{C})$ at the 226th position; $\mathrm{Y}^{d}$ indicates the additive nucleotide $\mathrm{Y}(\mathrm{C}>\mathrm{T})$ at the 122 nd position.

\subsection{Molecular Phylogenetic Tree Based on the ITS Gene Sequences}

A molecular phylogenetic tree based on ITS was generated as shown in Figure 2. A total amount of 13 nucleotide sequences, including 9 studied specimens and 4 pure lines from GenBank, were adopted to analyze the evolutionary relationship. 


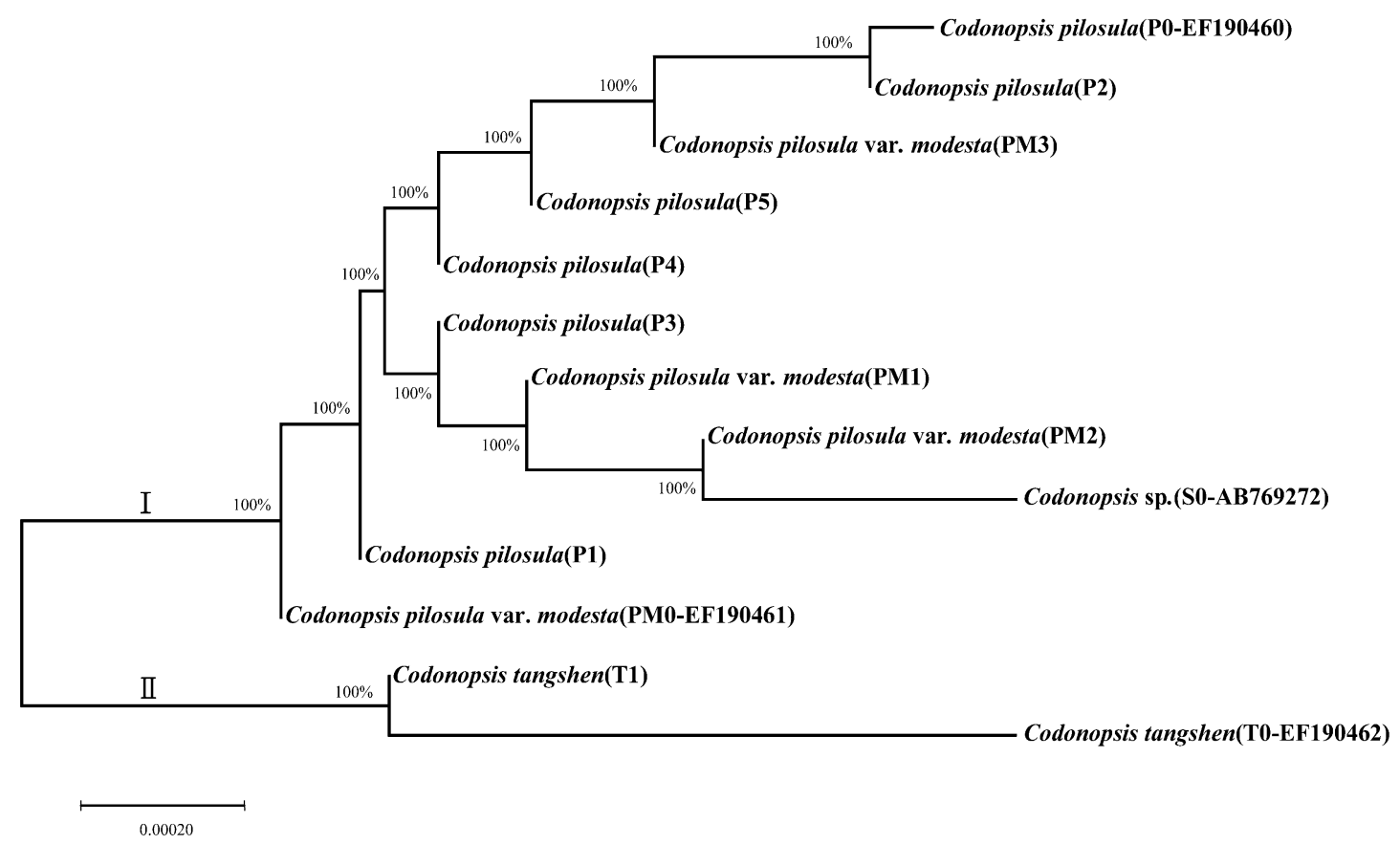

Figure 2. Neighbor-joining (NJ) evolutionary relationships of Codonopsis taxa based on internal transcribed spacer (ITS) sequences.

The studied specimens were highly homologous to the four pure lines from GenBank (Figure 2). Two monophyletic clades (designated as I and II) on the phylogenetic tree were developed. Clade I consisted of eight cultivars and three pure lines. The eight cultivars were composed of five ITS types of C. pilosula and three types of C. pilosula var. modesta. Clade II consisted of one cultivar of C. tangshen and one pure line. The phylogenetic tree in this study showed that C. pilosula, C. pilosula var. modesta, and $C$. tangshen were closely related, with a relatively closer relationship between the first two. C. tangshen had relatively far relationship with C. pilosula and C. pilosula var. modesta, which agreed with the definition in the Chinese Pharmacopeia. The unidentified Codonopsis sp. (S0) was closely related to C. pilosula var. modesta, rather than C. pilosula [14]. Accordingly, we speculated that it belonged to the species C. pilosula var. modesta.

\subsection{Hybridization Analysis Based on the ITS Gene Sequences}

According to the results of the aligned ITS sequence, all of the specimens in our investigation field were heterozygous. The additive nucleotide mainly presented at positions 122 and/or 226 in the species of C. pilosula and C. pilosula var. modesta. No pure line was found among the 45 specimens of Codonopsis Taxa. Combining the relationship in the phylogenetic tree with nucleotide additivity in the ITS sequence, the parental lineages could be referred [27]. The additive nucleotides at positions 122 and 226 were probably formed from a hybrid of two pure lines (Table 2). Five types (P1-P5) of ITS sequences were found in C. pilosula specimens, among which P1, P3, and P4 might be produced from the heterozygosis between P0 (C C) and S0 (T T), and P5 from P0 (C C) and PM0 (T C), and it was hard to speculate the hybrid origin of P2. Three types (PM1-PM3) of ITS sequences were determined in C. pilosula var. modesta, of which PM1 probably originated from the heterozygosis of $\mathrm{P0}$ (C C) and S0 (T T), PM2 from S0 (T T) and PM0 (T C), and PM3 from P0 (C C) and PM0 (T C).

The $S$-value was used to calculate the hybrid ratio of specimens, as expatiated in 2.1. The results in Table 3 show that among the 46 specimens, up to 33 of $C$. pilosula displayed sequence type $P 1$, and the average hybrid ratio at positions 122 and 226 were $39.01 \%$ and $33.69 \%$, respectively. The number of specimens with sequence type P2, P3, P4, and P5 were relatively small, only 1, 1, 2, and 2, respectively. The average hybrid ratio of type $\mathrm{P} 2$ at the 226th position was $33.5 \%$, and that of type $\mathrm{P} 3$ at positions 
122 and 226 was $32.7 \%$ and $48.4 \%$, respectively, and $36.32 \%$ and $24.52 \%$, respectively, for type P4. The heterozygosis of type 5 only happened at the 122 nd position, with an average hybrid ratio of $39.09 \%$. Six specimens of C. pilosula var. modesta were found and classified into three types. The average hybrid ratios of PM1 at positions 122 and 226 were $34.58 \%$ and 30.85\%, respectively, while that of PM2 at the 226th position was $25.53 \%$, and of PM3 at the 122 nd position was $27.35 \%$.

Table 3. The hybrid ratio of the genus Codonopsis specimens based on the ITS sequences $(n=3)$.

\begin{tabular}{|c|c|c|c|c|}
\hline Voucher No. & Species & $\begin{array}{c}\text { Sequence Type } \\
\text { (ITS) }\end{array}$ & $\begin{array}{c}\text { Hybrid Ratio } \\
(\%, 122 \text { nd })\end{array}$ & $\begin{array}{c}\text { Hybrid Ratio } \\
(\%, 226 \text { th })\end{array}$ \\
\hline CP01 & C. pilosula & P1 & $33.46 \pm 1.29$ & $46.89 \pm 1.35$ \\
\hline СР02 & C. pilosula & $\mathrm{P} 2$ & & $33.49 \pm 1.55$ \\
\hline СР03 & C. pilosula & P1 & $37.47 \pm 1.47$ & $37.07 \pm 0.50$ \\
\hline СР04 & C. pilosula & P1 & $36.14 \pm 1.73$ & $39.39 \pm 1.17$ \\
\hline СР05 & C. pilosula & P1 & $48.33 \pm 1.56$ & $25.14 \pm 2.52$ \\
\hline СР06 & C. pilosula & P1 & $35.23 \pm 1.31$ & $39.18 \pm 1.52$ \\
\hline СР07 & C. pilosula & P1 & $33.25 \pm 0.70$ & $41.59 \pm 0.45$ \\
\hline СР08 & C. pilosula & P1 & $44.77 \pm 1.09$ & $39.70 \pm 1.62$ \\
\hline СР09 & C. pilosula & P1 & $31.9 \pm 1.17$ & $33.57 \pm 2.13$ \\
\hline CP10 & C. pilosula & P3 & $32.72 \pm 1.48$ & $47.79 \pm 1.77$ \\
\hline CP11 & C. pilosula & P1 & $40.86 \pm 1.71$ & $29.05 \pm 2.04$ \\
\hline CP12 & C. pilosula & P1 & $38.34 \pm 1.26$ & $39.92 \pm 2.39$ \\
\hline CP13 & C. pilosula & P1 & $35.94 \pm 0.98$ & $40.33 \pm 3.32$ \\
\hline CP14 & C. pilosula & P1 & $44.52 \pm 0.72$ & $38.14 \pm 1.68$ \\
\hline CP15 & C. pilosula & P1 & $35.05 \pm 1.40$ & $43.61 \pm 2.30$ \\
\hline CP16 & C. pilosula & P1 & $30.65 \pm 0.99$ & $32.98 \pm 2.03$ \\
\hline CP17 & C. pilosula & P1 & $32.57 \pm 0.80$ & $28.64 \pm 0.82$ \\
\hline CP18 & C. pilosula & P1 & $45.63 \pm 0.93$ & $32.35 \pm 2.46$ \\
\hline CP19 & C. pilosula & P1 & $39.53 \pm 3.48$ & $40.20 \pm 2.11$ \\
\hline CP20 & C. pilosula & P1 & $32.24 \pm 0.63$ & $37.79 \pm 1.06$ \\
\hline $\mathrm{CP} 21$ & C. pilosula & P1 & $40.25 \pm 0.21$ & $40.62 \pm 1.27$ \\
\hline CP22 & C. pilosula & P1 & $35.93 \pm 0.35$ & $37.99 \pm 1.30$ \\
\hline $\mathrm{CP} 23$ & C. pilosula & P1 & $39.33 \pm 0.94$ & $33.61 \pm 1.03$ \\
\hline CP24 & C. pilosula & P1 & $36.48 \pm 0.57$ & $34.09 \pm 1.12$ \\
\hline CP25 & C. pilosula & P1 & $39.65 \pm 0.17$ & $34.66 \pm 0.83$ \\
\hline CP26 & C. pilosula & P4 & $47.64 \pm 1.72$ & $32.05 \pm 2.11$ \\
\hline СР27 & C. pilosula & P1 & $34.86 \pm 1.51$ & $22.40 \pm 1.51$ \\
\hline CP28 & C. pilosula & P1 & $43.02 \pm 0.83$ & $31.94 \pm 0.82$ \\
\hline СР29 & C. pilosula & P1 & $40.71 \pm 1.63$ & $25.37 \pm 1.88$ \\
\hline СР30 & C. pilosula & P1 & $48.02 \pm 0.41$ & $22.07 \pm 1.46$ \\
\hline CP31 & C. pilosula & P1 & $43.49 \pm 0.46$ & $20.11 \pm 1.74$ \\
\hline CP32 & C. pilosula & P1 & $47.36 \pm 0.41$ & $17.41 \pm 1.73$ \\
\hline CP33 & C. pilosula & P1 & $28.92 \pm 0.49$ & $29.00 \pm 5.90$ \\
\hline CP34 & C. pilosula & P5 & $34.88 \pm 5.96$ & \\
\hline CP35 & C. pilosula & P1 & $46.22 \pm 6.07$ & $28.87 \pm 1.41$ \\
\hline CP36 & C. pilosula & P1 & $43.10 \pm 4.92$ & $29.25 \pm 2.70$ \\
\hline CP37 & C. pilosula & P5 & $39.67 \pm 6.60$ & \\
\hline CP38 & C. pilosula & P4 & $24.14 \pm 0.85$ & $16.47 \pm 0.66$ \\
\hline CP39 & C. pilosula & P1 & $34.67 \pm 1.01$ & $42.17 \pm 0.71$ \\
\hline CPM40 & C. pilosula var. modesta & PM1 & $36.99 \pm 4.01$ & $38.67 \pm 0.54$ \\
\hline CPM41 & C. pilosula var. modesta & PM1 & $39.29 \pm 0.25$ & $33.92 \pm 1.33$ \\
\hline CPM42 & C. pilosula var. modesta & PM1 & $36.88 \pm 0.49$ & $21.23 \pm 0.66$ \\
\hline CPM43 & C. pilosula var. modesta & PM1 & $22.94 \pm 0.80$ & $19.67 \pm 0.95$ \\
\hline CPM44 & C. pilosula var. modesta & PM2 & & $25.56 \pm 1.40$ \\
\hline CPM45 & C. pilosula var. modesta & PM3 & $27.35 \pm 1.01$ & \\
\hline
\end{tabular}


Further analyses on the sequence type of $C$. pilosula and C. pilosula var. modesta manifested that types P1 (Y Y) and PM1 (Y Y) accounted for a large proportion, and the average hybrid ratios at positions 122 and 226 varied greatly, which suggested that multiple hybrids probably happened, rather than the hybrid of homozygotes P0 (C C) and S0 (T T). For example, S0 (T T) hybridized with PM0 (T C) to form PM2 (T Y), which probably further hybridized with P0 (C C) to form P1 (Y Y) or PM1 (Y Y). Additionally, PM3 (Y C) was produced from the hybrid between P0 (C C) and PM0 (T C), which could further hybridize with S0 (T T) to form P1 (Y Y) or PM1 (Y Y).

No obvious correlation between the heterozygous rate and geographical factors, including altitude longitude and latitude (Table 4 and Figure 3), could be speculated. Therefore, this phenomenon might arise from the selection of germplasm resources, which became more monotonous in long-term artificial cultivation. It is reported that a large number of variable nucleotide sites existed in the wild C. pilosula [42], so much more attention should be paid to the reasonable exploitation and genetic breeding of Codonopsis taxa.

Table 4. Cultivated Codonopsis species in this study.

\begin{tabular}{|c|c|c|c|c|c|c|}
\hline $\begin{array}{l}\text { Voucher } \\
\text { No. }\end{array}$ & Species & Locality & $\begin{array}{l}\text { Locality } \\
\text { No. }^{a}\end{array}$ & $\begin{array}{l}\text { Altitude } \\
(\mathrm{m})\end{array}$ & $\begin{array}{l}\text { Date of } \\
\text { Collection }\end{array}$ & $\begin{array}{c}\text { Sequence } \\
\text { Type (ITS) }^{b}\end{array}$ \\
\hline СР01 & C. pilosula & $\begin{array}{c}\text { Liancai, Longde, Guyuan, } \\
\text { Ningxia, China }\end{array}$ & 1 & 1760 & 20 July 2016 & P1 \\
\hline СР02 & C. pilosula & $\begin{array}{l}\text { Kezhai, Longxi, Dingxi, } \\
\text { Gansu, China }\end{array}$ & 2 & 2220 & $\begin{array}{l}25 \text { October } \\
2016\end{array}$ & P2 \\
\hline СР03 & C. pilosula & $\begin{array}{c}\text { Xiaozhai, Minxian, } \\
\text { Dingxi, Gansu, China }\end{array}$ & 3 & 2550 & $\begin{array}{l}25 \text { October } \\
2016\end{array}$ & P1 \\
\hline СР04 & C. pilosula & $\begin{array}{l}\text { Weixin, Minxian, Dingxi, } \\
\text { Gansu, China }\end{array}$ & 4 & 2225 & $\begin{array}{l}25 \text { October } \\
2016\end{array}$ & P1 \\
\hline СР05 & C. pilosula & $\begin{array}{l}\text { Xijiang, Minxian, Dingxi, } \\
\text { Gansu, China }\end{array}$ & 5 & 2254 & $\begin{array}{l}25 \text { October } \\
2016 \\
\end{array}$ & P1 \\
\hline СР06 & C. pilosula & $\begin{array}{l}\text { Meichuan, Minxian, } \\
\text { Dingxi, Gansu, China }\end{array}$ & 6 & 2328 & $\begin{array}{l}25 \text { October } \\
2016\end{array}$ & P1 \\
\hline СР07 & C. pilosula & $\begin{array}{l}\text { Minyang, Minxian, } \\
\text { Dingxi, Gansu, China }\end{array}$ & 7 & 2305 & $\begin{array}{l}25 \text { October } \\
2016\end{array}$ & P1 \\
\hline CP27 & C. pilosula & $\begin{array}{l}\text { Minyang, Minxian, } \\
\text { Dingxi, Gansu, China }\end{array}$ & & 2305 & $\begin{array}{l}14 \text { October } \\
2017\end{array}$ & P1 \\
\hline СР08 & C. pilosula & $\begin{array}{l}\text { Chabu, Minxian, Dingxi, } \\
\text { Gansu, China }\end{array}$ & 8 & 2313 & $\begin{array}{l}25 \text { October } \\
2016\end{array}$ & P1 \\
\hline CP28 & C. pilosula & $\begin{array}{l}\text { Mazichuan, Minxian, } \\
\text { Dingxi, Gansu, China }\end{array}$ & 9 & 2510 & $\begin{array}{l}14 \text { October } \\
2017\end{array}$ & P1 \\
\hline СР09 & C. pilosula & $\begin{array}{l}\text { Zhongzhai, Minxian, } \\
\text { Dingxi, Gansu, China }\end{array}$ & 10 & 2381 & $\begin{array}{l}25 \text { October } \\
2016\end{array}$ & P1 \\
\hline CP10 & C. pilosula & $\begin{array}{c}\text { Hadapu, Tanchang, } \\
\text { Longnan, Gansu, China }\end{array}$ & 11 & 2281 & $\begin{array}{l}28 \text { October } \\
2016\end{array}$ & P3 \\
\hline CP11 & C. pilosula & $\begin{array}{c}\text { Hadapu, Tanchang, } \\
\text { Longnan, Gansu, China }\end{array}$ & & 2250 & $\begin{array}{l}28 \text { October } \\
2016\end{array}$ & P1 \\
\hline CP12 & C. pilosula & $\begin{array}{c}\text { Hadapu, Tanchang, } \\
\text { Longnan, Gansu, China }\end{array}$ & & 2238 & $\begin{array}{l}28 \text { October } \\
2016\end{array}$ & P1 \\
\hline CP13 & C. pilosula & $\begin{array}{c}\text { Hadapu, Tanchang, } \\
\text { Longnan, Gansu, China }\end{array}$ & & 2233 & $\begin{array}{l}28 \text { October } \\
2016\end{array}$ & P1 \\
\hline CP14 & C. pilosula & $\begin{array}{c}\text { Hadapu, Tanchang, } \\
\text { Longnan, Gansu, China }\end{array}$ & & 2271 & $\begin{array}{l}28 \text { October } \\
2016\end{array}$ & P1 \\
\hline СР34 & C. pilosula & $\begin{array}{l}\text { Hadapu, Tanchang, } \\
\text { Longnan, Gansu, China }\end{array}$ & & 2445 & $\begin{array}{l}14 \text { October } \\
2017\end{array}$ & P4 \\
\hline CP35 & C. pilosula & $\begin{array}{l}\text { Hadapu, Tanchang, } \\
\text { Longnan, Gansu, China }\end{array}$ & & 2435 & $\begin{array}{l}14 \text { October } \\
2017\end{array}$ & P1 \\
\hline
\end{tabular}


Table 4. Cont.

\begin{tabular}{|c|c|c|c|c|c|c|}
\hline $\begin{array}{l}\text { Voucher } \\
\text { No. }\end{array}$ & Species & Locality & $\begin{array}{l}\text { Locality } \\
\text { No. }{ }^{a}\end{array}$ & $\begin{array}{l}\text { Altitude } \\
\text { (m) }\end{array}$ & $\begin{array}{c}\text { Date of } \\
\text { Collection }\end{array}$ & $\begin{array}{c}\text { Sequence } \\
\text { Type (ITS) }{ }^{b}\end{array}$ \\
\hline CP36 & C. pilosula & $\begin{array}{c}\text { Hadapu, Tanchang, } \\
\text { Longnan, Gansu, China }\end{array}$ & & 2188 & $\begin{array}{l}14 \text { October } \\
2017\end{array}$ & P1 \\
\hline CP37 & C. pilosula & $\begin{array}{c}\text { Hadapu, Tanchang, } \\
\text { Longnan, Gansu, China }\end{array}$ & & 2242 & $\begin{array}{l}14 \text { October } \\
2017\end{array}$ & P3 \\
\hline CP15 & C. pilosula & $\begin{array}{l}\text { Awu, Tanchang, Longnan, } \\
\text { Gansu, China }\end{array}$ & 12 & 2421 & $\begin{array}{l}28 \text { October } \\
2016\end{array}$ & P1 \\
\hline CP32 & C. pilosula & $\begin{array}{l}\text { Awu, Tanchang, Longnan, } \\
\text { Gansu, China }\end{array}$ & & 2351 & $\begin{array}{l}14 \text { October } \\
2017\end{array}$ & P1 \\
\hline CP33 & C. pilosula & $\begin{array}{l}\text { Awu, Tanchang, Longnan, } \\
\text { Gansu, China }\end{array}$ & & 2329 & $\begin{array}{l}14 \text { October } \\
2017\end{array}$ & P1 \\
\hline CP16 & C. pilosula & $\begin{array}{c}\text { Pangjia, Tanchang, } \\
\text { Longnan, Gansu, China }\end{array}$ & 13 & 2503 & $\begin{array}{l}28 \text { October } \\
2016\end{array}$ & P1 \\
\hline CP17 & C. pilosula & $\begin{array}{c}\text { Pangiia, Tanchang, } \\
\text { Longnan, Gansu, China }\end{array}$ & & 2368 & $\begin{array}{l}28 \text { October } \\
2016\end{array}$ & P1 \\
\hline CP18 & C. pilosula & $\begin{array}{c}\text { Pangiia, Tanchang, } \\
\text { Longnan, Gansu, China }\end{array}$ & & 2390 & $\begin{array}{l}28 \text { October } \\
2016\end{array}$ & P1 \\
\hline CP30 & C. pilosula & $\begin{array}{c}\text { Pangiia, Tanchang, } \\
\text { Longnan, Gansu, China }\end{array}$ & & 2431 & $\begin{array}{l}14 \text { October } \\
2017\end{array}$ & P1 \\
\hline CP31 & C. pilosula & $\begin{array}{c}\text { Pangiia, Tanchang, } \\
\text { Longnan, Gansu, China }\end{array}$ & & 2456 & $\begin{array}{l}14 \text { October } \\
2017\end{array}$ & P1 \\
\hline CP38 & C. pilosula & $\begin{array}{c}\text { Pangjia, Tanchang, } \\
\text { Longnan, Gansu, China }\end{array}$ & & 2320 & $\begin{array}{l}14 \text { October } \\
2017\end{array}$ & P5 \\
\hline CP39 & C. pilosula & $\begin{array}{c}\text { Pangiia, Tanchang, } \\
\text { Longnan, Gansu, China }\end{array}$ & & 2306 & $\begin{array}{l}14 \text { October } \\
2017\end{array}$ & P1 \\
\hline CP19 & C. pilosula & $\begin{array}{c}\text { Lichuan, Tanchang, } \\
\text { Longnan, Gansu, China }\end{array}$ & 14 & 2255 & $\begin{array}{l}28 \text { October } \\
2016\end{array}$ & P1 \\
\hline СР29 & C. pilosula & $\begin{array}{c}\text { Lichuan, Tanchang, } \\
\text { Longnan, Gansu, China }\end{array}$ & & 2255 & $\begin{array}{l}14 \text { October } \\
2017\end{array}$ & P1 \\
\hline CP20 & C. pilosula & $\begin{array}{c}\text { Lichuan, Tanchang, } \\
\text { Longnan, Gansu, China }\end{array}$ & & 2286 & $\begin{array}{l}28 \text { October } \\
2016\end{array}$ & P1 \\
\hline CP21 & C. pilosula & $\begin{array}{c}\text { Lichuan, Tanchang, } \\
\text { Longnan, Gansu, China }\end{array}$ & & 2388 & $\begin{array}{l}28 \text { October } \\
2016\end{array}$ & P1 \\
\hline CP22 & C. pilosula & $\begin{array}{c}\text { Lichuan, Tanchang, } \\
\text { Longnan, Gansu, China }\end{array}$ & & 2475 & $\begin{array}{l}28 \text { October } \\
2016\end{array}$ & P1 \\
\hline $\mathrm{CP} 23$ & C. pilosula & $\begin{array}{l}\text { Lichuan, Tanchang, } \\
\text { Longnan, Gansu, China }\end{array}$ & & 2314 & $\begin{array}{l}28 \text { October } \\
2016\end{array}$ & P1 \\
\hline CP24 & C. pilosula & $\begin{array}{c}\text { Lichuan, Tanchang, } \\
\text { Longnan, Gansu, China }\end{array}$ & & 2320 & $\begin{array}{l}28 \text { October } \\
2016\end{array}$ & P1 \\
\hline CP25 & C. pilosula & $\begin{array}{c}\text { Guanzhuang, Yaozhou, } \\
\text { Tongchuan, Shaanxi, } \\
\text { China }\end{array}$ & 15 & 880 & $\begin{array}{c}15 \\
\text { November } \\
2016\end{array}$ & P1 \\
\hline CP26 & C. pilosula & $\begin{array}{l}\text { Hongtiguan, Pingshun, } \\
\text { Changzhi, Shanxi, China }\end{array}$ & 16 & 1245 & $\begin{array}{l}12 \text { March } \\
2017\end{array}$ & P4 \\
\hline СРM40 & $\begin{array}{l}\text { C. pilosula } \\
\text { var. } \\
\text { modesta }\end{array}$ & $\begin{array}{c}\text { Danbao, Wenxian, } \\
\text { Longnan, Gansu, China }\end{array}$ & 17 & 895 & $\begin{array}{l}15 \text { October } \\
2016\end{array}$ & PM1 \\
\hline CPM41 & $\begin{array}{l}\text { C. pilosula } \\
\text { var. } \\
\text { modesta }\end{array}$ & $\begin{array}{c}\text { Koutouba, Wenxian, } \\
\text { Longnan, Gansu, China }\end{array}$ & 18 & 1266 & $\begin{array}{l}15 \text { October } \\
2016\end{array}$ & PM1 \\
\hline
\end{tabular}


Table 4. Cont.

\begin{tabular}{|c|c|c|c|c|c|c|}
\hline $\begin{array}{l}\text { Voucher } \\
\text { No. }\end{array}$ & Species & Locality & $\begin{array}{l}\text { Locality } \\
\text { No. }{ }^{a}\end{array}$ & $\begin{array}{l}\text { Altitude } \\
(\mathrm{m})\end{array}$ & $\begin{array}{c}\text { Date of } \\
\text { Collection }\end{array}$ & $\begin{array}{l}\text { Sequence } \\
\text { Type (ITS) }{ }^{b}\end{array}$ \\
\hline СРM42 & $\begin{array}{c}\text { C. pilosula } \\
\text { var. } \\
\text { modesta }\end{array}$ & $\begin{array}{c}\text { Shifang, Wenxian, } \\
\text { Longnan, Gansu, China }\end{array}$ & 19 & 995 & $\begin{array}{l}28 \text { October } \\
2016\end{array}$ & PM2 \\
\hline СРM43 & $\begin{array}{l}\text { C. pilosula } \\
\text { var. } \\
\text { modesta }\end{array}$ & $\begin{array}{c}\text { Baoziba, Wenxian, } \\
\text { Longnan, Gansu, China }\end{array}$ & 20 & 1634 & $\begin{array}{l}28 \text { October } \\
2016\end{array}$ & PM2 \\
\hline СРM44 & $\begin{array}{l}\text { C. pilosula } \\
\text { var. } \\
\text { modesta }\end{array}$ & $\begin{array}{c}\text { Baoziba, Wenxian, } \\
\text { Longnan, Gansu, China }\end{array}$ & & 1480 & $\begin{array}{l}28 \text { October } \\
2016\end{array}$ & PM3 \\
\hline CPM45 & $\begin{array}{l}\text { C. pilosula } \\
\text { var. } \\
\text { modesta }\end{array}$ & $\begin{array}{l}\text { Zhongzhai, Wenxian, } \\
\text { Longnan, Gansu, China }\end{array}$ & 21 & 1361 & $\begin{array}{l}28 \text { October } \\
2016\end{array}$ & PM2 \\
\hline CT46 & C. tangshen & $\begin{array}{l}\text { Banqiao, Enshi, Enshi, } \\
\text { Hubei, China }\end{array}$ & 22 & 1775 & $\begin{array}{l}18 \text { August } \\
2016\end{array}$ & $\mathrm{~T} 1$ \\
\hline
\end{tabular}

${ }^{a}$ Localities of collection are shown in Figure $3 ;^{b}$ the sequence type is indicated in Table 2.

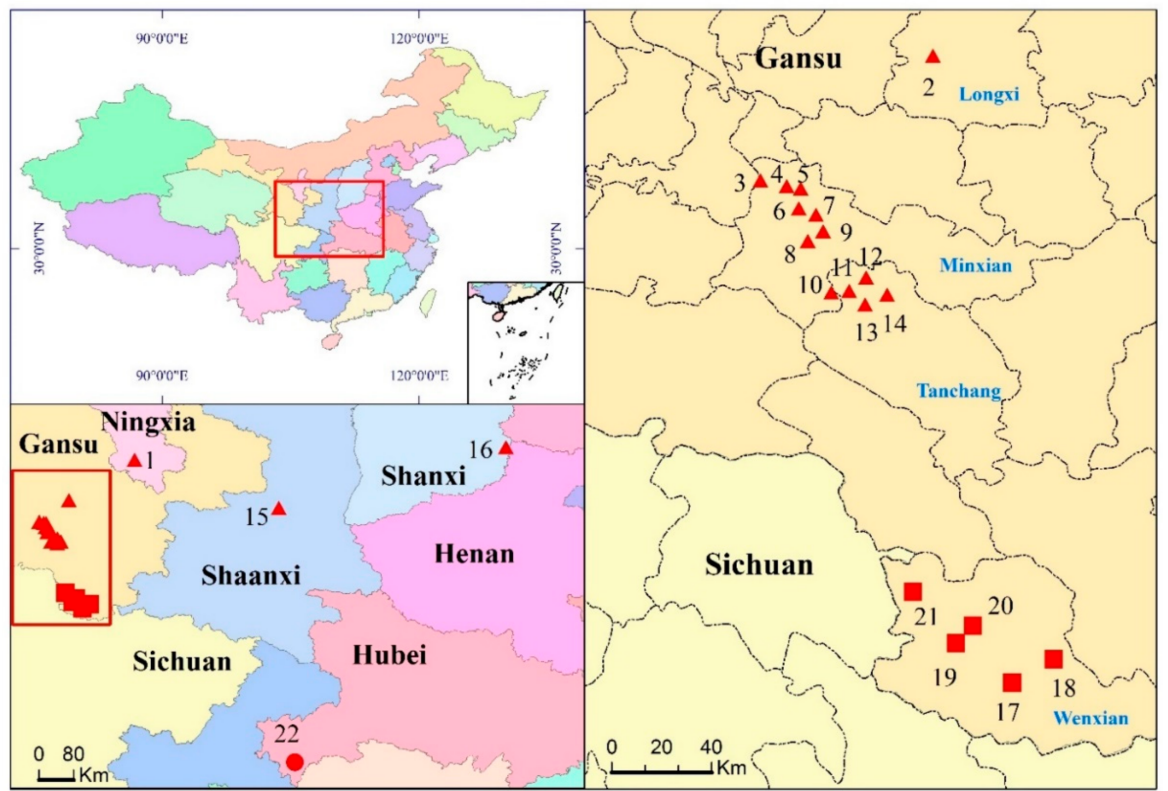

Figure 3. Collection sites of Codonopsis specimens during in 2016 and 2017. The numerals of the collection sites are indicated in Table 3. The solid triangles indicate C. pilosula. The solid squares indicate C. pilosula var. modesta. The solid circulars indicate C. tangshen.

\section{Materials and Methods}

\subsection{Plant Materials}

Forty-six identified Codonopsis specimens including 39 strains of C. pilosula, 6 strains of C. pilosula var. modesta, and 1 strain of $C$. tangshen were studied. They were collected from the cultivation fields of Gansu, Shanxi, Shaanxi, and Hubei province, and Ningxia Hui Autonomous Region of China during our field investigation from 2016 to 2017 (shown in Table 4). All specimens were stored in the laboratory of plant secondary metabolism and regulation of Zhejiang Province, College of Life Sciences, Zhejiang Sci-Tech University, China. 


\subsection{DNA Extraction}

The genomic DNA was extracted from 50-60 mg dried roots by Plant Genprep DNA Kit (Zoman Biotech Co., Beijing, China) with minor modifications to the protocol provided by manufacturer, that is, 5-6 mg polyvinyl pyrrolidone K40 (Molecular Biology Grade, Sangon Bio-tech Co., Shanghai, China) was mixed with the dried root before grinding. Incubation time at $65^{\circ} \mathrm{C}$ was extended from $30 \mathrm{~min}$ to $6 \mathrm{~h}$ [5]. The purity and quantity of extracted DNA was detected using NanoDrop-2000 (Thermo Scientific, Wilmington, NC, USA). The quality was detected by electrophoresis on $1.0 \%$ agarose gel stained with ethidium bromide. DNA samples were stored at $-20^{\circ} \mathrm{C}$ before using in the PCR amplification. Three replicates were prepared for DNA extraction and PCR amplification.

\subsection{PCR Amplification}

The primers used for amplification of ITS were oligonucleotide ITS5F (Forward primer 5'-GGA AGT AAA AGT CGT AAC AAG G-3') and ITS4R (Reverse primer $5^{\prime}$-TCC TCC GCT TAT TGA TAT GC-3') $[3,5,43]$. Amplification reaction was performed in a volume of $20 \mu \mathrm{L}$ mixture, including $10 \mu \mathrm{L}$ $2 \times$ Ftaq PCR Mix (Zoman Bio-technology Co., Beijing, China), $0.25 \mu \mathrm{M}$ of each primer, approximate 20-100 ng template DNA, and $8 \mu \mathrm{L}$ RNase-free water. A T100 thermal cycler (Bio-Rad Laboratories, Inc., Hercules, CA, USA) was used to carry out PCR amplification under the cycling condition: initial denaturation at $95^{\circ} \mathrm{C}$ for $5 \mathrm{~min}$, followed by 35 cycles of denaturation at $95^{\circ} \mathrm{C}$ for $30 \mathrm{~s}$, annealing at $50^{\circ} \mathrm{C}$ for $30 \mathrm{~s}$, extension at $72{ }^{\circ} \mathrm{C}$ for $50 \mathrm{~s}$, and then final extension at $72{ }^{\circ} \mathrm{C}$ for $5 \mathrm{~min}$. The $5 \mu \mathrm{L}$ of PCR product was detected by $1.0 \%$ agarose gel electrophoresis and sequencing was performed by Tsingke Bio-tech Co., Hangzhou, China. The primers in sequencing and PCR amplification were the same and two directional sequencing were conducted using ABI 3730XL sequencer (SeqGen, Inc., Torrance, CA, USA).

\subsection{Verification on Variable Nucleotide}

In ITS sequencing of Codonopsis specimens, double peaks were found in one position of the base, which might be produced from variable nucleotides. To confirm the presence of variable nucleotides, verification experiments were conducted referring to the previous studies with minor adjustments $[17,19]$. According to the ITS sequencing results of the 46 specimens, CP02 and CT46 were selected for the verification of variable nucleotide, because they had a single cytosine (C) and thymidine (T) peak at the 122th position, respectively. The ITS gene of the two specimens was amplified and the concentration of PCR products was detected. A series of PCR mixtures were prepared with the ratio of CT46 10\%, 15\%, 20\%,30\%, 40\%, and 50\%, respectively, and five replicates for each mixture. All PCR samples were sequenced by Qingke Biotechnology Co., Hangzhou, China. Same primers were used for PCR amplification and sequencing as 3.3. The sequencing results were examined using the Bioedit program [44]. The relative intensities of the secondary peak $(S)$ and the background noise level $(N)$ at the 122nd position were manually calculated as follows.

$$
\begin{aligned}
& S-\text { value }=\frac{I_{2}}{I_{1}+I_{2}} \\
& N-\text { value }=\frac{I_{0}}{I_{0}+I_{1}}
\end{aligned}
$$

where $I_{0}$ is the intensity of noise peak, which is the average intensity of peaks from the 117th to 127th nucleotide sites; $I_{1}$ is the intensity of the main peak at the 122 nd nucleotide site; and $I_{2}$ is the intensity of the secondary peak at the 122 nd nucleotide site.

\subsection{Processing on ITS Sequence}

The two directional sequences obtained by sequencing were assembled and edited using the ContigExpress program [45]. The nucleotide composition of the sequences was then analyzed and variable base sites were recorded. The presence of additive nucleotide is confirmed as follows. 
The possible positions of additive nucleotides in ITS sequences were inspected. If the same positions are found in the forward and reverse sequences, $S$ and $N$ values will be calculated based on the forward sequences using the Bioedit program [44]. If a significant difference exists in the two values, the presence of additive nucleotides can be confirmed.

According to the reported nucleotide composition of the ITS (ITS1-5.8S-ITS2) sequence $[5,16,19]$, it was found that the initial base of ITS contained "TCGAA" and the terminal base contained "TCCGACC", and the $5.8 \mathrm{~S}$ region began with "AAACGACTCT" and ended at "CGTCACGC". Thus, the ITS gene sequence can be obtained, and the length of ITS, ITS1, 5.8S, and ITS2 can be calculated.

The BLAST tool was used to compare and analyze the related data of the sequence and NCBI website. Based on the ITS gene sequences, the phylogenetic tree was analyzed on hierarchical clustering of the ITS alignments, and produced by MEGA 7 program [46] with neigbour-joining of the bootstrap values (1000 replicates).

\section{Conclusions}

This study optimized the method of direct sequencing of mixed PCR products to detect additive nucleotides of $C$. pilosula, $C$. pilosula var. modesta, and C. tangshen in the nrDNA ITS sequence. When the ratio of PCR product added up to $15 \%$, the presence of additive nucleotides could be confirmed. Compared with Codonopsis specimens collected during the year 2009 and 2010, the genetic pure lines disappeared, the ITS type reduced greatly, and the variable base sites declined from six to two in the specimens collected during the year 2016 to 2017. These phenomena show that the genetic diversity of Codonopsis taxa in main production areas has degenerated. Therefore, measures should be taken to build germplasm resources of Codonopsis taxa, protecting the genetic diversity and promoting the sustainable development of the Codonopsis taxa industry. The results of the ITS sequencing also indicated that the ITS marker alone could not be used to identify the three Codonopsis taxa, other morphological classification and molecular marker technologies should be combined to identify them.

Supplementary Materials: The following are available online at http:/ /www.mdpi.com/1420-3049/23/7/1565/ s1, Figure S1: The thumbnail picture of ITS sequences of 46 Codonopsis specimens.

Author Contributions: Conceptualization, L.-J.L. and Z.-S.L.; Formal analysis, L.-J.L. and B.-C.X.; Funding acquisition, F.L.; Investigation, L.-J.L., E.-H.W., Y.-C.Y., B.-C.X., W.J., F.L., and Z.-S.L.; Methodology, Z.-S.L.; Project administration, L.-J.L., F.L., and Z.-S.L.; Resources, E.-H.W. and Y.-C.Y.; Software, L.-J.L.; Supervision, Z.-S.L.; Validation, B.-C.X.; Writing—original draft, L.-J.L.; Writing—review \& editing, W.J. and Z.-S.L.

Funding: This research was funded by the 13th five-year plan of Ministry of Science and Technology of the People's Republic of China (2015BAC-01B-03).

Conflicts of Interest: The authors declare that they have no conflict of interest.

\section{References}

1. Commission, C.P. Pharmacopoeia of the People's Republic of China; China Medical Science Press: Beijing, China, 2015; Volume I, pp. 281-282. ISBN 978-7-5067-7337-9.

2. Wang, J.; Deng, C.-Q.; Shi, L.; Deng, M.-L. Progress of modern research on Codonopsis taxa. Guide China Med. 2011, 9, 279-281. [CrossRef]

3. Liu, X.-X.; Chen, Y.-L.; Shi, Y.-T.; Luo, J.-J.; Hou, Y.-Q.; Zhang, Y.-P. Analysis on genetic diversity of germplasm resources of radix Codonopsis cultivated in gansu province based on ISSR marker. J. Chin. Med. Mater. 2016, 39, 1742-1747. [CrossRef]

4. Yang, H.-Z.; Chen, Y.; Guo, F.-X.; Zhou, C.-M.; He, Y.-L. Resource investigation of wild Codonopsis pilosula inTanchang county of Gansu. China J. Chin. Mater. Med. 2016, 41, 186-191. [CrossRef]

5. Zhao, S.; Xin, T.-Y.; Hou, D.-Y.; Pang, X.-H.; Chen, R.-Y.; Gao, J.-P. Identification of Codonopsis Radix and Its Adulterants Using the ITS/ITS2 Barcodes. World Sci. Technol./Mod. Tradit. Chin. Med. Mater. Med. 2013, 15, 421-428. [CrossRef] 
6. Liu, D.; Guo, H.-Y.; Tang, W.-Q.; Yang, J.-Q. Comparative Evolution of S7 Intron 1 and Ribosomal Internal Transcribed Spacer in Coilia nasus (Clupeiformes: Engraulidae). Int. J. Mol. Sci. 2012, 13, 3085-3100. [CrossRef] [PubMed]

7. Shu, Z.-M.; Wang, Z.; Mu, X.-Q.; Liang, Z.-S.; Guo, H.-B. A Dominant Gene for Male Sterility in Salvia miltiorrhiza Bunge. PLoS ONE 2012, 7, e50903. [CrossRef] [PubMed]

8. Das, A.; Nandagopal, K.; Jha, T.B. Molecular characterization of some Indian Aloe vera populations through RAPD and ITS markers. Plant Biosyst. 2017, 151, 695-703. [CrossRef]

9. Han, E.-H.; Lee, S.-J.; Kim, M.-B.; Shin, Y.-W.; Kim, Y.-H.; Lee, S.-W. Molecular marker analysis of Cynanchum wilfordii and C-auriculatum using the simple ARMS-PCR method with mismatched primers. Plant Biotechnol. Rep. 2017, 11, 127-133. [CrossRef]

10. Jia, Q.-J.; Wang, J.-M.; Zhu, J.-H.; Hua, W.; Shang, Y.; Yang, J.-M.; Liang, Z.-S. Toward Identification of Black Lemma and Pericarp Gene Blp1 in Barley Combining Bulked Segregant Analysis and Specific-Locus Amplified Fragment Sequencing. Front. Plant Sci. 2017, 8, 1414. [CrossRef] [PubMed]

11. Mao, R.-J.; Xia, P.-G.; He, Z.-G.; Liu, Y.; Liu, F.-H.; Zhao, H.-G.; Han, R.-L.; Liang, Z.-S. Identification of seeds based on molecular markers and secondary metabolites in Senna obtusifolia and Senna occidentalis. Bot. Stud. 2017, 58, 43-52. [CrossRef] [PubMed]

12. Sun, W.; Yan, S.; Li, J.-J.; Xiong, C.; Shi, Y.-H.; Wu, L.; Xiang, L.; Deng, B.; Ma, W.; Chen, S. Study of Commercially Available Lobelia chinensis Products Using Bar-HRM Technology. Front. Plant Sci. 2017, 8, 1-7. [CrossRef] [PubMed]

13. Wang, X.-Y.; Chen, X.-C.; Yang, P.; Wang, L.-L.; Han, J.-P. Barcoding the Dendrobium (Orchidaceae) Species and Analysis of the Intragenomic Variation Based on the Internal Transcribed Spacer 2. Biomed. Res. Int. 2017, 2017, 1-10. [CrossRef] [PubMed]

14. Jiao, J.; Jia, X.-R.; Liu, P.; Zhang, Q.-M.; Liu, F.; Ma, C.-D.; Xi, P.-Z.; Liang, Z.-S. Species identification of polygonati rhizoma in China by both morphological and molecular marker methods. Comptes Rendus Biol. 2018, 341, 102-110. [CrossRef] [PubMed]

15. Mao, R.-J.; Xia, P.-G.; Liu, J.-L.; Li, X.; Han, R.-L.; Liu, F.-H.; Zhao, H.-G.; Liang, Z.-S. Genetic diversity and population structure assessment of Chinese Senna obtusifolia L. by molecular markers and morphological traits of seed. Acta Physiol. Plant. 2018, 40, 12. [CrossRef]

16. Lin, T.-C.; Hsieh, C.C.; Agrawal, D.C.; Kuo, C.-L.; Chue, F.-S.; Tsay, H.S. ITS sequence based phylogenetic relationship of Dangshen radix. J. Food Drug Anal. 2007, 15, 428-432. [CrossRef]

17. Kitani, Y.; Zhu, S.; Batkhuu, J.; Sanchir, C.; Komatsu, K. Genetic diversity of Ephedra plants in mongolia inferred from internal transcribed spacer sequence of nuclear ribosomal DNA. Biol. Pharm. Bull. 2011, 34, 717-726. [CrossRef] [PubMed]

18. Peng, H.-S.; Yuan, Q.-J.; Li, Q.-Q.; Huang, L.-Q. Molecular Systematics of Genus Atractylodes (Compositae, Cardueae): Evidence from Internal Transcribed Spacer (ITS) and trnL-F Sequences. Int. J. Mol. Sci. 2012, 13, 14623-14633. [CrossRef] [PubMed]

19. He, J.-Y.; Zhu, S.; Komatsu, K.; Goda, Y.; Cai, S.-Q. Genetic polymorphism of medicinally-used Codonopsis species in an internal transcribed spacer sequence of nuclear ribosomal DNA and its application to authenticate Codonopsis Radix. J. Nat. Med. 2014, 68, 112-124. [CrossRef] [PubMed]

20. Guo, L.-C.; Zhao, M.-M.; Sun, W.; Teng, H.-L.; Huang, B.-S.; Zhao, X.-P. Differentiation of the Chinese minority medicinal plant genus Berchemia spp. by evaluating three candidate barcodes. Springerplus 2016, 5, 658. [CrossRef] [PubMed]

21. Han, E.-H.; Cho, K.; Goo, Y.; Kim, M.; Shin, Y.-W.; Kim, Y.-H.; Lee, S.-W. Development of molecular markers, based on chloroplast and ribosomal DNA regions, to discriminate three popular medicinal plant species, Cynanchum wilfordii, Cynanchum auriculatum, and Polygonum multiflorum. Mol. Biol. Rep. 2016, 43, 323-332. [CrossRef] [PubMed]

22. Xia, P.-G.; Guo, H.-B.; Zhang, Y.; Deyholos, M.K.; Peng, L.; Jia, Y.-Y.; Yan, X.-J.; Liu, Y.; Liang, Z.-S. Wild Panax vietnamensis and Panax stipuleanatus markedly increase the genetic diversity of Panax notoginseng (Araliaceae) revealed by start codon targeted (SCoT) markers and ITS DNA barcode. Biochem. Syst. Ecol. 2016, 66, 37-42. [CrossRef]

23. Baldwin, B.G. Phylogenetic utility of the internal transcribed spacers of nuclear ribosomal DNA in plants: An example from the compositae. Mol. Phylogenetics Evol. 1992, 1, 3-16. [CrossRef] 
24. Baldwin, B.G.; Sanderson, M.J.; Porter, J.M.; Wojciechowski, M.F. The ITS Region of Nuclear Ribosomal DNA: A Valuable Source of Evidence on Angiosperm Phylogeny. Ann. Mo. Bot. Gard. 1995, 82, 247-277. [CrossRef]

25. Denk, T.; Grimm, G.W. Phylogeny and biogeography of Zelkova (Ulmaceae sensu stricto) as inferred from leaf morphology, ITS sequence data and the fossil record. Bot. J. Linn. Soc. 2005, 147, 129-157. [CrossRef]

26. Calonje, M.; Martín-Bravo, S.; Dobeš, C.; Koch, M.A. Non-coding nuclear DNA markers in phylogenetic reconstruction. Plant Syst. Evol. 2009, 282, 257-280. [CrossRef]

27. Sang, T.; Crawford, D.J.; Stuessy, T.F. Documentation of reticulate evolution in peonies (Paeonia) using internal transcribed spacer sequences of nuclear ribosomal DNA: Implications for biogeography and concerted evolution. Proc. Natl. Acad. Sci. USA 1995, 92, 6813-6817. [CrossRef] [PubMed]

28. Emshwiller, E.; Doyle, J. Origins of domestication and polyploidy in oca (Oxalis tuberosa: Oxalidaceae): NrDNA ITS data. Am. J. Bot. 1998, 85, 975-985. [CrossRef] [PubMed]

29. Franzke, A.; Mummenhoff, K. Recent hybrid speciation in Cardamine(Brassicaceae)+conversion of nuclear ribosomal ITS sequences in statu nascendi. Theor. Appl. Genet. 1999, 98, 831-834. [CrossRef]

30. Widmer, A.; Baltisberger, M. Molecular evidence for allopolyploid speciation and a single origin of the narrow endemic Draba ladina (Brassicaceae). Am. J. Bot. 1999, 86, 1282-1289. [CrossRef] [PubMed]

31. Whittall, J.; Liston, A.; Gisler, S.; Meinke, R. Detecting nucelotide additivity from direct sequences is a SNAP: An example from Sidalcea (Malvaceae). Plant Biol. 2000, 2, 211-217. [CrossRef]

32. Jason, T.; Rauscher, J.; Doyle, J.J.; Brown, A.H.D. Internal transcribed spacer repeat-specific primers and the analysis of hybridization in the Glycine tomentella (Leguminosae) polyploid complex. Mol. Ecol. 2002, 11, 2691-2702. [CrossRef]

33. Feng, T.; Zhou, Z.; Tang, J.; Cheng, M.; Zhou, S. ITS sequence variation supports the hybrid origin of Malus toringoides hughes. Can. J. Bot 2007, 85, 659-666. [CrossRef]

34. Liao, L.; Xu, L.-L.; Zhang, D.-M.; Fang, L.; Deng, H.-S.; Shi, J.-W.; Li, T.-J. Multiple hybridization origin of Ranunculus cantoniensis $(4 \times)$ : Evidence from trnL-F and ITS sequences and fluorescent in situ hybridization (FISH). Plant Syst. Evol. 2008, 276, 31-37. [CrossRef]

35. Soltis, D.E.; Mavrodiev, E.V.; Doyle, J.J.; Rauscher, J.; Soltis, P.S. ITS and ETS sequence data and phylogeny reconstruction in allopolyploids and hybrids. Syst. Bot. 2008, 33, 7-20. [CrossRef]

36. Consaul, L.L.; Gillespie, L.J.; Waterway, M.J. Evolution and polyploid origins in north American arctic puccinellia (poaceae) based on nuclear ribosomal spacer and chloroplast DNA sequences. Am. J. Bot. 2010, 97, 324-336. [CrossRef] [PubMed]

37. Koch, M.A.; Karl, R.; German, D.A.; Al-Shehbaz, I.A. Systematics, taxonomy and biogeography of three new Asian genera of Brassicaceae tribe Arabideae: An ancient distribution circle around the Asian high mountains. Taxon 2012, 61, 955-969.

38. Punina, E.O.; Machs, E.M.; Krapivskaya, E.E.; Kim, E.-S.; Mordak, E.V.; Myakoshina, Y.A.; Rodionov, A.V. Interspecific hybridization in the genus Paeonia (Paeoniaceae): Polymorphic sites in transcribed spacers of the $45 \mathrm{~S}$ rRNA genes as indicators of natural and artificial peony hybrids. Russ. J. Genet. 2012, 48, 684-697. [CrossRef]

39. Mandakova, T.; Kovarik, A.; Zozomova-Lihova, J.; Shimizu-Inatsugi, R.; Shimizu, K.K.; Mummenhoff, K.; Marhold, K.; Lysak, M.A. The More the Merrier: Recent Hybridization and Polyploidy in Cardamine. Plant Cell 2013, 25, 3280-3295. [CrossRef] [PubMed]

40. Hodac, L.; Scheben, A.P.; Hojsgaard, D.; Paun, O.; Horandl, E. ITS Polymorphisms Shed Light on Hybrid Evolution in Apomictic Plants: A Case Study on the Ranunculus auricomus Complex. PLoS ONE 2014, 9, 1-18. [CrossRef] [PubMed]

41. Zozomova Lihova, J.; Krak, K.; Mandakova, T.; Shimizu, K.K.; Spaniel, S.; Vit, P.; Lysak, M.A. Multiple hybridization events in Cardamine (Brassicaceae) during the last 150 years: Revisiting a textbook example of neoallopolyploidy. Ann. Bot. 2014, 113, 817-830. [CrossRef] [PubMed]

42. Luo, H.-B.; Zhang, J.; Hu, Z.-H.; Yuan, D.-P. Molecular identification of wild and cultivated banqiao Codonopsis species. Lishizhen Med. Mater. Res. 2010, 21, 3272-3273. [CrossRef]

43. Li, D.-Z.; Gao, L.-M.; Li, H.-T.; Wang, H.; Ge, X.-J.; Liu, J.-Q.; Chen, Z.-D.; Zhou, S.-L.; Chen, S.-L.; Yang, J.-B. Comparative analysis of a large dataset indicates that internal transcribed spacer (ITS) should be incorporated into the core barcode for seed plants. Proc. Natl. Acad. Sci. USA 2011, 108, 19641-19646. [CrossRef] [PubMed]

44. Elkins, K.M. Chapter 15-Analysis of Deoxyribonucleic Acid (DNA) Sequence Data Using BioEdit. In Forensic DNA Biology; Academic Press: San Diego, CA, USA, 2013; pp. 129-132. 
45. Lu, G.-Q.; Moriyama, E.N. Vector NTI, a balanced all-in-one sequence analysis suite. Brief. Bioinform. 2004, 5, 378-388. [CrossRef] [PubMed]

46. Kumar, S.; Stecher, G.; Tamura, K. MEGA7: Molecular Evolutionary Genetics Analysis Version 7.0 for Bigger Datasets. Mol. Biol. Evol. 2016, 33, 1870-1874. [CrossRef] [PubMed]

Sample Availability: Samples of the Codonopsis taxa are available from the authors. 